COMMISSIONS G1 AND G4 OF THE IAU

INFORMATION BULLETIN ON VARIABLE STARS

Volume 63 Number 6206 DOI: 10.22444/IBVS.6206

Konkoly Observatory

Budapest

3 May 2017

HU ISSN $0374-0676$

\title{
MINIMA TIMES OF THREE SELECTED SYSTEMS IN CANCER
}

GÖKAY, G.; DERMAN, E.; GÜROL, B.

Ankara University, Faculty of Science, Dept. of Astronomy and Space Sciences, Ankara, TÜRKİY; e-mail: ggokay@science.ankara.edu.tr

\section{Observatory and telescope:}

37" Kepler Space Telescope

\begin{tabular}{|l|l|}
\hline Detector: & 42 e2v CCD90s cameras, total 105 square degree FOV, \\
& $2200 \times 1024$ pixels for each CCD \\
\hline
\end{tabular}

\begin{tabular}{|l|}
\hline Method of data reduction: \\
\hline Data used here are pre-search data conditioning simple aperture photometry flux \\
values and downloaded from Keplen 1 archive. \\
\hline
\end{tabular}

\section{Method of minimum determination:}

All minima times are weighted average BJD of the values obtained with parabolic and sine function fitting and Kwee \& van Woerden (1956) method.

\footnotetext{
${ }^{1}$ https://archive.stsci.edu/k2/
} 


\begin{tabular}{|c|c|c|c|c|c|}
\hline \multicolumn{6}{|c|}{ Times of minima: } \\
\hline Star name & $\begin{array}{l}\text { Time of min. } \\
\text { HJD 2400000+ }\end{array}$ & Error & Type & Filter & Rem. \\
\hline ES Cnc & 57140.19210 & 0.00016 & II & Kepler & \\
\hline & 57140.72375 & 0.00025 & I & Kepler & \\
\hline & 57141.25920 & 0.00027 & II & Kepler & \\
\hline & 57141.79312 & 0.00714 & $\mathrm{I}$ & Kepler & \\
\hline & 57142.32820 & 0.00023 & II & Kepler & \\
\hline & 57142.86174 & 0.00023 & I & Kepler & \\
\hline & 57143.39817 & 0.00029 & II & Kepler & \\
\hline & 57143.92864 & 0.00113 & I & Kepler & \\
\hline & 57144.46591 & 0.00019 & II & Kepler & \\
\hline & 57144.99722 & 0.00028 & I & Kepler & \\
\hline & 57145.53485 & 0.00031 & II & Kepler & \\
\hline & 57146.06515 & 0.00029 & I & Kepler & \\
\hline & 57146.60330 & 0.00018 & II & Kepler & \\
\hline & 57147.13166 & 0.00026 & $\mathrm{I}$ & Kepler & \\
\hline & 57147.67304 & 0.00022 & II & Kepler & \\
\hline & 57148.20084 & 0.00048 & I & Kepler & \\
\hline & 57148.74229 & 0.00041 & II & Kepler & \\
\hline & 57149.26815 & 0.00030 & I & Kepler & \\
\hline & 57149.80610 & 0.00098 & II & Kepler & \\
\hline & 57150.33772 & 0.00029 & I & Kepler & \\
\hline & 57150.87567 & 0.00028 & II & Kepler & \\
\hline & 57151.40554 & 0.00602 & I & Kepler & \\
\hline & 57151.94642 & 0.00073 & II & Kepler & \\
\hline & 57152.47549 & 0.00646 & I & Kepler & \\
\hline & 57153.01687 & 0.00057 & II & Kepler & \\
\hline & 57153.54085 & 0.00020 & I & Kepler & \\
\hline & 57154.08282 & 0.00049 & II & Kepler & \\
\hline & 57154.60948 & 0.00766 & I & Kepler & \\
\hline & 57155.14936 & 0.00050 & II & Kepler & \\
\hline & 57155.67797 & 0.00453 & I & Kepler & \\
\hline & 57156.21496 & 0.00038 & II & Kepler & \\
\hline & 57156.74506 & 0.00026 & I & Kepler & \\
\hline & 57157.28206 & 0.00020 & II & Kepler & \\
\hline & 57157.81205 & 0.00016 & I & Kepler & \\
\hline & 57158.35135 & 0.00034 & II & Kepler & \\
\hline & 57158.87954 & 0.00216 & I & Kepler & \\
\hline & 57159.42186 & 0.00050 & II & Kepler & \\
\hline & 57159.94770 & 0.00552 & I & Kepler & \\
\hline & 57160.48549 & 0.00015 & II & Kepler & \\
\hline & 57161.01506 & 0.00063 & I & Kepler & \\
\hline & 57161.55666 & 0.00097 & II & Kepler & \\
\hline & 57162.08280 & 0.00011 & $\mathrm{I}$ & Kepler & \\
\hline & 57162.62475 & 0.00046 & II & Kepler & \\
\hline & 57163.15032 & 0.00034 & I & Kepler & \\
\hline & 57163.69094 & 0.00029 & II & Kepler & \\
\hline & 57164.21934 & 0.00189 & $\mathrm{I}$ & Kepler & \\
\hline & 57164.75272 & 0.00022 & II & Kepler & \\
\hline
\end{tabular}




\begin{tabular}{|c|c|c|c|c|c|}
\hline \multicolumn{6}{|c|}{ Times of minima: } \\
\hline Star name & $\begin{array}{l}\text { Time of min. } \\
\text { HJD } 2400000+\end{array}$ & Error & Type & Filter & Rem. \\
\hline ES Cnc & 57165.28665 & 0.00435 & $\mathrm{I}$ & Kepler & \\
\hline & 57165.82562 & 0.00027 & II & Kepler & \\
\hline & 57166.35503 & 0.00032 & I & Kepler & \\
\hline & 57166.89236 & 0.00057 & II & Kepler & \\
\hline & 57167.42170 & 0.00032 & $\mathrm{I}$ & Kepler & \\
\hline & 57167.95407 & 0.00010 & II & Kepler & \\
\hline & 57168.48991 & 0.00030 & I & Kepler & \\
\hline & 57169.02107 & 0.00033 & II & Kepler & \\
\hline & 57169.55777 & 0.00420 & I & Kepler & \\
\hline & 57170.09457 & 0.00029 & II & Kepler & \\
\hline & 57170.62625 & 0.00050 & I & Kepler & \\
\hline & 57171.16488 & 0.00036 & II & Kepler & \\
\hline & 57171.69359 & 0.00027 & I & Kepler & \\
\hline & 57172.22657 & 0.00006 & II & Kepler & \\
\hline & 57172.75957 & 0.00045 & $\mathrm{I}$ & Kepler & \\
\hline & 57173.29561 & 0.00032 & II & Kepler & \\
\hline & 57173.82863 & 0.00022 & I & Kepler & \\
\hline & 57174.36198 & 0.00026 & II & Kepler & \\
\hline & 57174.89761 & 0.00031 & $\mathrm{I}$ & Kepler & \\
\hline & 57175.42997 & 0.00055 & II & Kepler & \\
\hline & 57175.96378 & 0.00017 & I & Kepler & \\
\hline & 57176.49622 & 0.00050 & II & Kepler & \\
\hline & 57177.03168 & 0.00022 & I & Kepler & \\
\hline & 57177.56336 & 0.00057 & II & Kepler & \\
\hline & 57178.09863 & 0.00024 & I & Kepler & \\
\hline & 57178.62873 & 0.00063 & II & Kepler & \\
\hline & 57179.16728 & 0.00021 & $\mathrm{I}$ & Kepler & \\
\hline & 57179.69860 & 0.00055 & II & Kepler & \\
\hline & 57180.23340 & 0.00018 & $\mathrm{I}$ & Kepler & \\
\hline & 57180.76379 & 0.00069 & II & Kepler & \\
\hline & 57181.29939 & 0.00030 & I & Kepler & \\
\hline & 57181.83277 & 0.00024 & II & Kepler & \\
\hline & 57182.36647 & 0.00019 & $\mathrm{I}$ & Kepler & \\
\hline & 57182.90359 & 0.00023 & II & Kepler & \\
\hline & 57183.43470 & 0.00018 & I & Kepler & \\
\hline & 57183.97141 & 0.00054 & II & Kepler & \\
\hline & 57184.50271 & 0.00037 & I & Kepler & \\
\hline & 57185.03389 & 0.00080 & II & Kepler & \\
\hline & 57185.57243 & 0.00037 & $\mathrm{I}$ & Kepler & \\
\hline & 57186.09937 & 0.00046 & II & Kepler & \\
\hline & 57186.64222 & 0.00073 & I & Kepler & \\
\hline & 57187.16963 & 0.00058 & II & Kepler & \\
\hline & 57187.70618 & 0.00029 & I & Kepler & \\
\hline & 57188.23707 & 0.00098 & II & Kepler & \\
\hline & 57188.77556 & 0.00025 & $\mathrm{I}$ & Kepler & \\
\hline & 57189.30662 & 0.00167 & II & Kepler & \\
\hline & 57189.84304 & 0.00035 & $\mathrm{I}$ & Kepler & \\
\hline
\end{tabular}




\begin{tabular}{|c|c|c|c|c|}
\hline \multicolumn{5}{|c|}{ Times of minima: } \\
\hline Star name & $\begin{array}{l}\text { Time of min. } \\
\text { HJD 2400000+ }\end{array}$ & Error & Type & Filter \\
\hline \multirow[t]{44}{*}{ ES Cnc } & 57190.37177 & 0.00026 & II & Kepler \\
\hline & 57190.91064 & 0.00079 & I & Kepler \\
\hline & 57191.44585 & 0.00052 & II & Kepler \\
\hline & 57191.97891 & 0.00023 & I & Kepler \\
\hline & 57192.52141 & 0.00095 & II & Kepler \\
\hline & 57193.57799 & 0.00056 & II & Kepler \\
\hline & 57194.11157 & 0.00071 & I & Kepler \\
\hline & 57194.65228 & 0.00030 & II & Kepler \\
\hline & 57195.17933 & 0.00148 & I & Kepler \\
\hline & 57195.72360 & 0.00088 & II & Kepler \\
\hline & 57196.24820 & 0.00039 & I & Kepler \\
\hline & 57196.78810 & 0.00073 & II & Kepler \\
\hline & 57197.31624 & 0.00071 & $\mathrm{I}$ & Kepler \\
\hline & 57197.85499 & 0.00026 & II & Kepler \\
\hline & 57198.38547 & 0.00036 & $\mathrm{I}$ & Kepler \\
\hline & 57198.92577 & 0.00048 & II & Kepler \\
\hline & 57199.45250 & 0.00040 & I & Kepler \\
\hline & 57199.98996 & 0.00040 & II & Kepler \\
\hline & 57200.52199 & 0.00060 & $\mathrm{I}$ & Kepler \\
\hline & 57201.06137 & 0.00034 & II & Kepler \\
\hline & 57201.59063 & 0.00038 & I & Kepler \\
\hline & 57202.12900 & 0.00073 & II & Kepler \\
\hline & 57202.65799 & 0.00034 & $\mathrm{I}$ & Kepler \\
\hline & 57203.19854 & 0.00088 & II & Kepler \\
\hline & 57203.72754 & 0.00011 & I & Kepler \\
\hline & 57204.26789 & 0.00094 & II & Kepler \\
\hline & 57204.79494 & 0.00250 & I & Kepler \\
\hline & 57205.33497 & 0.00046 & II & Kepler \\
\hline & 57205.86145 & 0.00032 & I & Kepler \\
\hline & 57206.39606 & 0.00074 & II & Kepler \\
\hline & 57206.92953 & 0.00084 & I & Kepler \\
\hline & 57207.47214 & 0.00042 & II & Kepler \\
\hline & 57207.99790 & 0.00046 & I & Kepler \\
\hline & 57208.54200 & 0.00058 & II & Kepler \\
\hline & 57209.06401 & 0.00104 & I & Kepler \\
\hline & 57209.60887 & 0.00079 & II & Kepler \\
\hline & 57210.13328 & 0.00029 & I & Kepler \\
\hline & 57210.68092 & 0.00077 & II & Kepler \\
\hline & 57211.20089 & 0.00113 & $\mathrm{I}$ & Kepler \\
\hline & 57211.74893 & 0.00034 & II & Kepler \\
\hline & 57212.26886 & 0.00045 & I & Kepler \\
\hline & 57212.81547 & 0.00086 & II & Kepler \\
\hline & 57213.33577 & 0.00031 & I & Kepler \\
\hline & 57213.88478 & 0.00046 & II & Kepler \\
\hline
\end{tabular}




\begin{tabular}{|lllcl|}
\hline \multicolumn{2}{|l}{ Times of minima: } & & & \\
\hline Star name & Time of min. & Error & Type & Filter \\
& HJD 2400000+ & & & \\
\hline HV Cnc & 57144.49617 & 0.00647 & II & Kepler \\
& 57149.70916 & 0.00469 & I & Kepler \\
& 57154.87854 & 0.02173 & II & Kepler \\
& 57160.05590 & 0.00071 & I & Kepler \\
& 57165.22350 & 0.00170 & II & Kepler \\
& 57170.39350 & 0.00023 & I & Kepler \\
& 57175.56557 & 0.00444 & II & Kepler \\
& 57180.73304 & 0.00239 & I & Kepler \\
& 57191.07121 & 0.00061 & I & Kepler \\
& 57201.41008 & 0.00033 & I & Kepler \\
& 57206.57681 & 0.00119 & II & Kepler \\
& 57211.74497 & 0.00304 & I & Kepler \\
HD 75638 & 57141.93059 & 0.00050 & I & Kepler \\
& 57153.56623 & 0.00082 & I & Kepler \\
& 57159.38222 & 0.00100 & I & Kepler \\
& 57165.20106 & 0.00032 & I & Kepler \\
57171.01750 & 0.00945 & I & Kepler \\
57194.28592 & 0.01062 & I & Kepler \\
57205.92409 & 0.00060 & I & Kepler \\
\hline
\end{tabular}

\section{Acknowledgements:}

This paper includes data collected by the Kepler/K2 mission. Funding for the Kepler/K2 mission is provided by the NASA Science Mission directorate.

Reference:

Kwee, K. K., van Woerden, H., 1956, Bull. Astron. Inst. Neth., 12, 327 